\title{
Universal hierarchical symmetry for turbulence and general multi-scale fluctuation systems
}

\author{
Zhen-Su She • Zhi-Xiong Zhang
}

Received: 9 April 2009 / Accepted: 20 April 2009 / Published online: 19 May 2009

(C) The Author(s) 2009. This article is published with open access at Springerlink.com

\begin{abstract}
Scaling is an important measure of multi-scale fluctuation systems. Turbulence as the most remarkable multi-scale system possesses scaling over a wide range of scales. She-Leveque (SL) hierarchical symmetry, since its publication in 1994, has received wide attention. A number of experimental, numerical and theoretical work have been devoted to its verification, extension, and modification. Application to the understanding of magnetohydrodynamic turbulence, motions of cosmic baryon fluids, cosmological supersonic turbulence, natural image, spiral turbulent patterns, DNA anomalous composition, human heart variability are just a few among the most successful examples. A number of modified scaling laws have been derived in the framework of the hierarchical symmetry, and the SL model parameters are found to reveal both the organizational order of the whole system and the properties of the most significant fluctuation structures. A partial set of work related to these studies are reviewed. Particular emphasis is placed on the nature of the hierarchical symmetry. It is suggested that the SL hierarchical symmetry is a new form of the self-organization principle for multi-scale fluctuation systems, and can be employed as a standard analysis tool in the general multi-scale methodology. It is further suggested that the SL hierarchical symmetry implies the existence of a turbulence ensemble. It is speculated that the search for defining the turbulence ensemble might open a new way for deriving statistical closure equations for turbulence and other multi-scale fluctuation systems.
\end{abstract}

The project supported by the National Natural Science Foundation (90716008) and by MOST 973 project (2009CB724100).

Z.-S. She $(\varangle) \cdot$ Z.-X. Zhang

State Key Laboratory for Turbulence and Complex Systems, College of Engineering, Peking University, 100871 Beijing, China e-mail: she@pku.edu.cn
Keywords Turbulence - Scaling law $\cdot$ She-Leveque model · Hierarchical symmetry $\cdot$ Self-organization · Turbulence ensemble

\section{Introduction}

In 1994, She and Leveque [1] made the following proposal: Fully developed turbulence may be characterized by a hierarchy of fluctuation events with increasing intensities, and these intensities are related to each other by a so-called hierarchical symmetry, and the scaling properties are then completely determined by the scaling of the most intermittent and intensive fluctuation structures. For homogenous isotropic incompressible turbulence, by assuming the filamentary structure for the most intermittent structure, it further yields a formula for the scaling exponents of the $p$ th-order velocity structure function:

$\zeta_{p}=p / 9+2\left[1-\left(\frac{2}{3}\right)^{p / 3}\right]$,

a formula known today as the She-Leveque (SL) scaling. Later, it is shown that the proposed scaling corresponds exactly to a random log-Poisson multiplicative process $[2,3]$, which stands as the simplest process with an infinitely divisible property. This is the property that is crucial for describing cascade as a stochastic process, so SL model is often called Log-Poisson model.

Over the past fifteen years, the SL scaling has received a widely spread attention; scholars from nearly forty nations have discussed this work; their related papers have appeared in more than one hundred different journals published all over the world. A number of research work have been accomplished, which range from verification in experimental 
measurements and numerical simulations to applications in explaining scaling in various physical systems. Many different formulas of scaling law have been achieved following SL theory (see Table 1). Are there common features among the systems to satisfy the SL hierarchical symmetry? How is the SL hierarchical symmetry analysis applied to such a variety of systems? These questions are addressed by the main body of the material reviewed in this article. However, we are always attracted to questions of more abstract form, such as why the SL hierarchical symmetry is universal, and how much it adds to our understanding of the nature of turbulence, and finally, what might be the next significant step in our theoretical description of turbulence. We attempt to discuss those questions as well, within our limited capability. The central point to make is that, with increasingly abundant experimental and numerical data, accurate model of turbulence should be attainable in the near future, if right theoretical concepts arise.

\section{The SL theory of hierarchical symmetry}

The SL theory starts with the definition of a hierarchy of fluctuation intensities. Let $\delta_{\ell} v$ denote the velocity increment across a distance $\delta_{\ell} v=v(x+\ell)-v(x)$ and $S_{p}(\ell)$ denote the velocity structure function, $S_{p}(\ell)=\left\langle\left|\delta_{\ell} \nu\right|^{p}\right\rangle$. The hierarchy is defined by

$F_{p}(\ell)=\frac{S_{p+1}(\ell)}{S_{p}(\ell)} ; \quad p=0,1,2, \ldots$.

The hierarchy covers a series of increasing intensities from the mean $\left(F_{0}=\left\langle\left|\delta_{\ell} v\right|\right\rangle\right)$ to the so-called most intermittent structures, $F_{\infty}(\ell)=\lim _{p \rightarrow \infty} F_{p}(\ell)$. Note that the hierarchy covers both a range of scales and a range of intensities. Kolmogorov [4] was the first to point out the importance of multiple scales and gave a linear law for scaling exponents, $\zeta_{p}=p / 3(\mathrm{~K} 41)$; this linear scaling law is known to depart from experimental results. The deviation is the well-known problem of anomalous scaling of intermittency effects. The SL theory emphasizes the integrity of multiple intensities together with the multi-scale property. This integrity is represented by a hierarchical symmetry assumption, hereafter referred to as the SL hierarchical symmetry:

$F_{p+1}(\ell)=A_{p} F_{p}(\ell)^{\beta} F_{\infty}(\ell)^{1-\beta}$

where $\beta \leqslant 1$ is a parameter and $A_{p}$ is independent of $\ell$. This assumption yields a general formula of the scaling exponents $\zeta_{p}$ of the velocity structure function, $S_{p} \sim \ell^{\zeta_{p}}$,

$\zeta_{p}=\gamma p+C\left(1-\beta^{p}\right)$

where $\gamma$ describes the singularity index of the most intermittent structure: $F_{\infty}(\ell) \sim \ell^{\gamma}$, and $C$ is its co-dimension.

Based on the assumption of the hierarchical symmetry (3), she et al. [5-7] proposed a procedure called " $\beta$-test" and
" $\gamma$-test" to determine the parameters $\beta$ and $\gamma$ in the general SL formula (4), since turbulence in different environment may have different SL parameters while the hierarchical symmetry still holds. Eliminating $F_{\infty}(\ell)^{1-\beta}$, one finds a scaling relation

$$
\frac{F_{p+1}(\ell)}{F_{2}(\ell)}=\frac{A_{p}}{A_{1}}\left(\frac{F_{p}(\ell)}{F_{1}(\ell)}\right)^{\beta},
$$

A log- $\log$ plot of $F_{p+1}(\ell) / F_{2}(\ell)$ versus $F_{p}(\ell) / F_{1}(\ell)$ with a straight line implies that the SL hierarchical symmetry holds, hence the data passed the $\beta$-test, the value of $\beta$ can be readily obtained from the slope, which measures how similar are the scaling of the two successive HS intensities $F_{p+1}(\ell)$ and $F_{p}(\ell)$. The limit $\beta \rightarrow 1$ implies the absence of intermittency, and all intensities are similar. This includes the Kolmogorov 1941 picture of turbulence with $\zeta_{p}=p / 3$. On the other hand, the limit $\beta \rightarrow 0$ implies that the most intermittent structures are extremely outstanding, and completely detached from the background fluctuations. The black and white picture of turbulence by the $\beta$ model [8] belongs to this category. A close example is Burgers shocks whose energy spectrum is $k^{-2}$ and whose scaling exponents are $\zeta_{p}=1$ for $p>1[9,10]$.

Assuming extended self-similarity (ESS) [11], a property that generally holds in multi-scale phenomena $\left(\zeta_{3}=1\right)$, one can show that the parameter $\gamma$ defined by $F_{\infty}(\ell) \sim \ell^{\gamma}$ is determined by the relation

$\zeta_{p}-\chi(p ; \beta)=\gamma(p-3 \chi(p ; \beta))$,

where $\chi(p ; \beta)=\left(1-\beta^{p}\right) /\left(1-\beta^{3}\right)$. The plot of $\zeta_{p}-\chi(p ; \beta)$ vs $p-3 \chi(p ; \beta)$ yields a slope $\gamma \cdot \gamma$ is a measure of the degree of singularity of the most intermittent structure $F_{\infty}$. If the most intermittent structures were discontinuities, $\gamma=0$. An example of a weaker singularity is a velocity profile like Brownian motion for which $\gamma=1 / 2$. The larger $\gamma$ is, the weaker the singularity of the most intermittent structure is. Kolmogorovs dissipative structure has $\gamma=1 / 3$, less singular than shocks. The SL model has smoothly made a bridge between the Kolmogorov turbulence and Burgers turbulence.

As a phenomenological theory, the SL model has two important features. First, the hierarchical symmetry assumption can be directly tested against the experimental and numerical data, because it is formulated in terms of statistical moments. This is in contrast with other random multiplicative cascade (RMC) models, for which in the RMC is already a strong assumption about the state of multi-scale fluctuation field. Many physical systems (as we will see below) have a limited range of excited scales and can hardly support a multi-stage cascade. Yet, the moments across scales develop the SL hierarchical symmetry. Hence, the SL hierarchical symmetry appears more fundamental than the cascade scenario. The second property of the model is its direct link, through the concept of the most intermittent structure, to the geometrical aspect of turbulent structures, which is 
very unique among all intermittency models of turbulence. This makes it particularly attractive as it is applied to study other systems.

According to Kadanoff [12], "the SL scaling gives partially empirical formulas for the probability of various sizes of velocity fluctuations, and has become an important part of the phenomenology of scaling". In his review of intermittency models, Sreenivasan [13] pointed out that most of the models can be best cast in terms of multifractal formalism, and the SL scaling model is "particularly interesting, popular, and potentially powerful". While a certain degree of popularity of the model is interesting, we are more attracted to its universal nature, the hierarchical symmetry aspect. In the following sections, we review its developments in several areas, including experimental and numerical studies, and the application to magnetohydrodynamic turbulence, highly compressible and supersonic turbulence in astrophysical environment and several other multi-scale systems. Finally, we offer a theoretical discussion on the nature of the hierarchical symmetry, namely the principle of selforganization in multi-scale fluctuation fields and the existence of the so-called turbulence ensemble.

\section{Experimental verifications of the hierarchical symmetry}

The publication of She and Leveque [1] has generated a great interest from experimental, numerical and theoretical studies on the hierarchical nature of turbulence. Several months after the publication of SL94, Ruiz Chavarria et al. [14] conducted experimental measurements in a wind tunnel for turbulence generated with grids, in a wake behind a cylinder and in a jet with Taylor microscale Reynolds numbers ranging from 200 to 800 , and concluded that the hierarchical structure assumption is valid for the fluctuation field of the energy dissipation in all experiments. A year later, the same authors [15] claimed that their data also confirm the existence of the hierarchical symmetry for the velocity structure functions. They [16] have also discovered that the temperature structure function displays a similar hierarchy. The following year, Camussi and Benzi [17] concluded that the SL prediction was realized in all cases, confirming that, within the experimental uncertainty, the geometrical interpretation of $\beta$ could be extended to the transverse velocity components (see also Benzi et al. $[18,19]$ for more details). Leveque et al. [20] observed, in a turbulent wake, that the moments of passive scalar energy diffusion rate satisfy a similar hierarchical symmetry relation. The evidences from all these classical experiments offer a solid basis for the validity of the SL hierarchical symmetry, and provides enough stimulation for continuous interests later on further checks, modifications and applications.

Swinney's group at the University of Texas at Austin has conducted experimental studies of turbulence in the
Couette-Taylor flow and a rapidly rotating tank with variable Rossiby numbers. The Couette-Taylor flow [24] reaches a Reynolds number range of $2 \times 10^{3}<R<10^{6}$ with no inertial range observed, but careful analysis of the velocity structure functions using a systematic method of $\beta$-test and $\gamma$-test [5], which is derived from the SL theory, showed that the SL hierarchical symmetry is verified to a high quality, and the SL parameter $\beta \approx 0.83$, slightly smaller than the value $\beta \approx 0.87$ in free jets. This yielded an accurate indication of greater intermittency in Couette-Taylor turbulence.

In 2003, Baroud et al. [23] made measurements of velocity time series and of velocity field in a rotating annulus for small Rossiby number of quasi two-dimensional flow to high Rossiby number of fully three-dimensional flow. Over the range of flows of different nature, the SL hierarchical symmetry is universally verified with different values of the SL parameters. While the hierarchical similarity parameter $\beta$ showed very little difference, the singularity index of the most intermittent structure $\gamma$ varies from 0.18 in 3D (close to isotropic turbulence) to 0.34 in $2 \mathrm{D}$. The latter showed a scaling close to Kolmogorov turbulence. See Fig. 1 for a sketch of the results.

Experimental studies were also conducted at the Peking University with a turbulent pipe flow and a turbulent mixing layer. Zou et al. [21] analyzed velocity fluctuation data in a pipe at a dozen distances from the wall ranging from 10 to 3000 in wall unit. They found that the SL hierarchical symmetry is verified for all data, but the parameters $\beta$ and $\gamma$

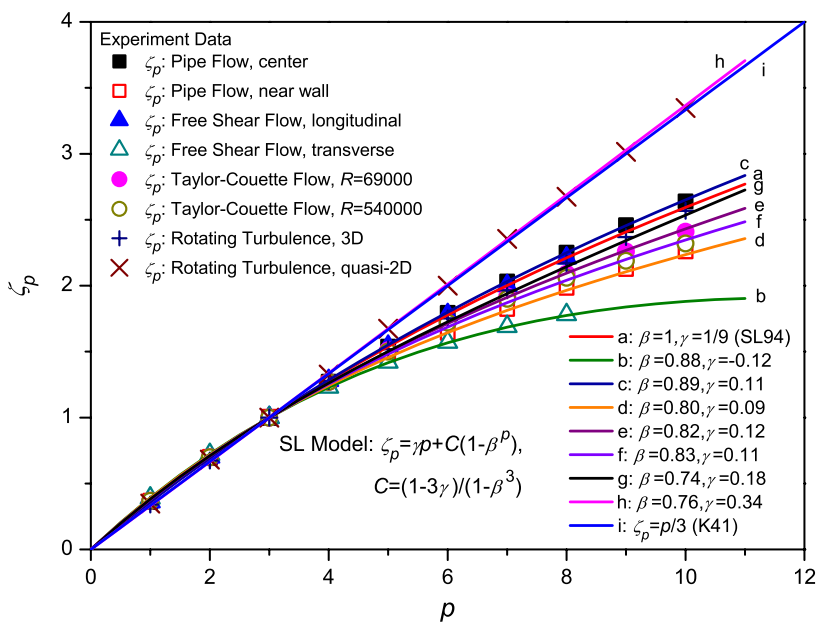

Fig. 1 Verification of the SL hierarchical symmetry by various turbulence experiments: $(\square)$ velocity measurement at the center of a pipe $(\square)$ near the wall of a pipe [21]; $(\mathbf{\Delta})$ longitudinal and $(\triangle)$ transverse velocity structure function measured in a free shear flow [22]; (•) velocity measurement in a Taylor-Couette flow at $R=69000$ and (o) at $R=540000$ from [5]; $(+)$ velocity measurements in rotating annulus at very small Rossiby number (a fully 3D flow) and $(x)$ at large Rossiby number (a quasi-2D flow) from [23]. Lines $a-h$ denote corresponding SL model with different parameters, all satisfying the SL hierarchical symmetry. Line $i$ is the $\mathrm{K} 41$ prediction 
vary with the distance from the wall. The variation of $\beta$ and $\gamma$ was shown to display a transition from the logarithmic region to the center, which is interpreted to be associated with the breakdown of large-scale organized structures.

Jiang et al. [22] conducted the simultaneous measurement of two velocity components in a turbulent mixing layer, which allows for the calculation of both longitudinal and transverse velocity structure functions. They showed that the hierarchical symmetry is universally valid for all the measurements, at several space locations, and for two different upstream turbulence generation mechanisms. Interestingly, the parameter $\beta$ specifying similarity between weak and strong vortices is shown to be universal for all structure functions, and the other parameter $\gamma$ of the singularity index of the most intermittent structures vary. The most singular structure is found to be related to the structure function associated with the stream wise vortices. The results confirm that coherent flow structures are responsible for anomalous scaling; hence, the deviation from the normal scaling can be an indicator of the development of flow structures and the SL parameters have a sound correspondence with characteristic flow structures.

A series of studies of Rayleigh-Bénard convection turbulence have been done at the Chinese University of Hong Kong (CUHK) by Xia's and Ching's group. Using experimental data of Libchaber at the University of Chicago [25,26], Ching and Kwok [27] found that local temperature dissipation in high Rayleigh number convection satisfy a similar SL hierarchy, and Ching [28] showed that the scaling behavior of the temperature structure functions for length scales below and above the Bolgiano scale are both well described by the SL hierarchical symmetry. Both velocity fluctuations [29] and temperature fluctuations [30] were confirmed to be welldescribed by the SL hierarchical symmetry. Xia et al. [31] made a direct multipoint measurements of the velocity and temperature fields, and showed that in the central region, velocity and temperature both have excellent SL scaling with different model parameters.

Parts of the experimental results are shown in Fig. 1. While the isotropic turbulence data for the longitudinal velocity structure function agree accurately with the original SL formula, the data for transverse velocity structure functions and for other inhomogeneous turbulence show the validity of the SL hierarchical symmetry but with different model parameters. It can be concluded that the experimental measurements give a firm verification of the SL hierarchical symmetry which is universally valid.

\section{Numerical verifications of the hierarchical symmetry}

During the past four decades, significant progress has been made using computer simulation as an aid to our understanding of the rich physics of turbulence, and numerical simulation now becomes an irreplaceable tool for the study of turbulence. Massive dataset enable more detailed and accurate statistical analysis. After the publication of the original work, a number of researchers have used their data to verify the hierarchical symmetry and the scaling exponents.

\subsection{Velocity fluctuations}

From a high-resolution direct numerical simulations (DNS) of 3D Navier-Stokes turbulence with normal viscosity and hyper viscosity, Cao et al. [32] found that relative scaling exponents are in very good agreement with the prediction of the SL model (see Fig. 2). Sreenivasan et al. [33] found that the low-order relative scaling exponents exhibit unambiguous departures from the Kolmogorov 1941 theory and agree very well with the SL model (see Fig. 2). Chen and Cao [34] have proposed a modified model within the framework of the SL hierarchical symmetry, which reads

$\zeta_{p}=\frac{2}{9}\left[1-\left(\frac{7}{9}\right)^{p / 3}\right]$.

Grossmann et al. [35] also conducted a study of intermittency for longitudinal and transversal structure functions in numerical simulations of the Navier-Stokes turbulence with $R e_{\lambda}=110$. They concluded that longitudinal and transversal fluctuations obey different types of hierarchies of the moments within the SL model, and the SL hierarchy

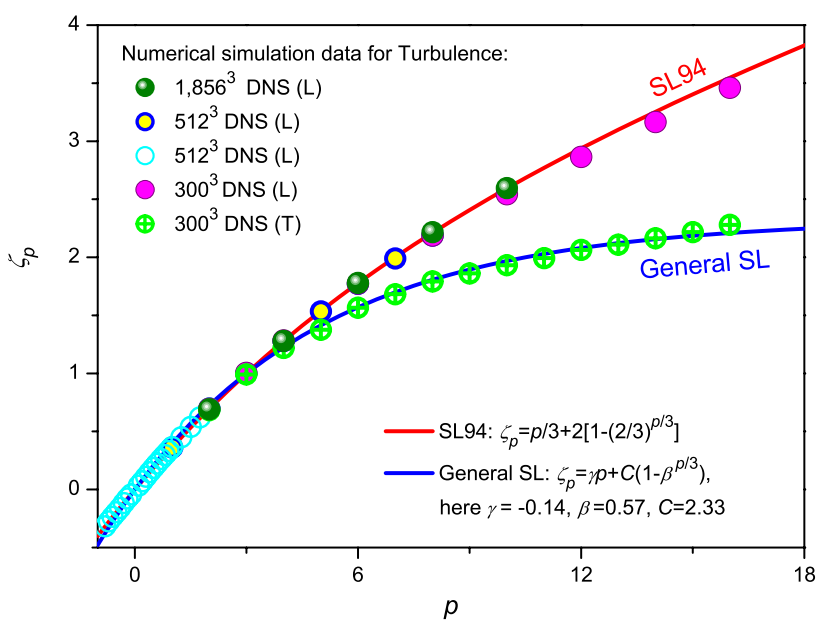

Fig. 2 Verification of the SL hierarchical symmetry by direct numerical simulations. Several sets of scaling exponents of longitudinal (L) and transversal $(\mathrm{T})$ velocity structure function are shown: (ball) terascale computation of weakly compressible turbulence using IBM Blue GeneL system with $1,856^{3}$ grids and 32,768 processors [36]; (yellow filled-o) $512^{3}$ simulation of incompressible NS turbulence $\left(R_{\lambda} \sim 181\right)$ from [32]; (o) low-order scaling exponents from [33]; (•) $300^{3} \mathrm{DNS}$ simulation of decaying turbulence $\left(R_{\lambda} \sim 250\right)$ from [37]; $(\oplus)$ scaling exponents of transverse velocity structure function from [38]. Two lines correspond to the origin SL model [1] and a general version of the SL model with parameters proposed in [3] 
parameter $\beta$ shows small dependency on the order of the moment. If credible, their results imply that the SL hierarchical symmetry holds only approximately. More studies are needed for clarifying this sensitive issue.

Boratav and Pelz [37] also carried out a detailed study on the difference between the longitudinal and transversal velocity structure functions using the direct numerical simulations of an unforced flow starting from a high-symmetry initial condition by Kida [39]. While comparisons to several intermittency models and experiments are carried out, special focus is given to the SL model. With freely adjusting parameters, the SL model gives good predictions for all the scaling values. Later, using his data at higher resolution $\left(300^{3}\right)$ and at higher Reynolds number $\left(R_{\lambda} \sim 250\right)$, Boratav [38] reported that the original SL model predictions are in very good agreement with longitudinal scaling exponents and the transverse scaling exponents deviate further away from the K41 scaling and they compare well with the SL model using $C_{0}=2.326$ and $\beta=0.57$. Additional discussion also can be found in [40]. All the results suggest that the SL hierarchical symmetry is universally valid for all the numerical data.

The most recent report comes from a group of scientists at the ASC FLASH center at the University of Chicago, who have produced the largest weakly compressible, homogeneous isotropic turbulence simulation to date, and obtained a dataset of very high quality. Among their initial scientific results has the clear claim: "analysis on the data show support for new theories of turbulent structure", namely the theory of the SL hierarchical symmetry and the SL scaling formula [36]. Specifically, they noted that: "As is easily seen, Kolmogorovs 1941 theoretical prediction deviates systematically from both Benzis experimental analysis and our simulation. In contrast, the more recent theory of She and Leveque is in close agreement with both experiment and simulation" (see Fig. 2).

\subsection{Passive scalar fluctuations}

Using the framework of the SL hierarchical symmetry, Leveque et al. [20] developed a model for the scaling of a mixed structure functions $\left\langle\left|\delta_{\ell} v \delta_{\ell} \theta^{2}\right|^{p}\right\rangle$ describing dynamics of passive scalar. Based on an assumption about sheet-like dissipative structures (see $[41,42]$ ), they predicted a scaling formula, which reads

$\Psi_{p}=\frac{p}{9}+\frac{10}{9}\left[1-\left(\frac{2}{5}\right)^{p / 3}\right]$.

When compared to experimental measurements from a turbulent wake behind a cylinder, they found excellent agreement(see Fig. 3). This prediction agrees also with the numerical results of Watanabe and Gotoh [43] of passive scalar statistics in homogeneous turbulence (see Fig. 3).

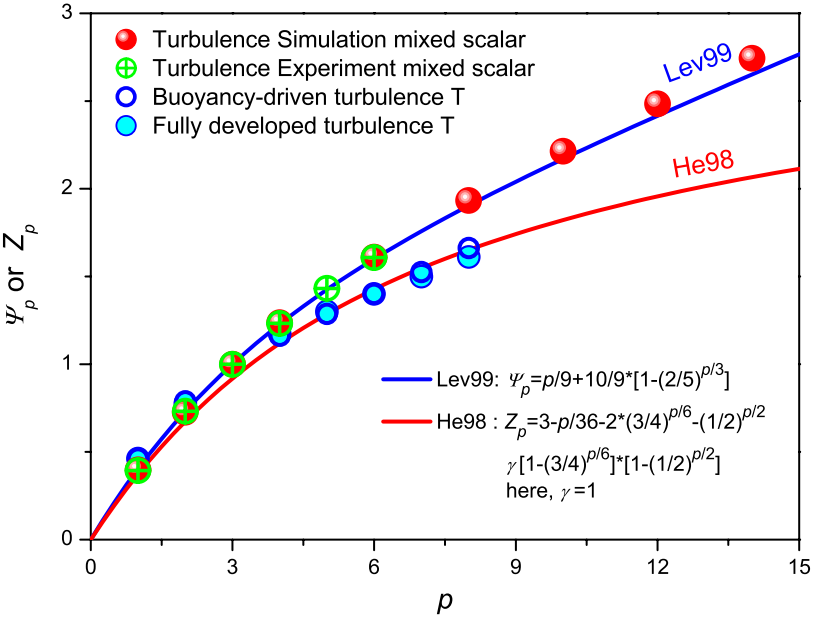

Fig. 3 Verification of the SL hierarchical symmetry for scaling of the passive scalar structure function: (ball) numerical scaling exponents for mixed velocity-scalar correlations in stationary homogeneous turbulence at $R_{\lambda}=427$ and $P_{\lambda}=427$ from high-resolution simulations [43]; $(\bigoplus)$ experimental scaling exponents for mixed velocity-scalar correlations in heated cylinder turbulence with $R_{\lambda} \simeq 300$ from [20]; (o) experimental scaling exponents for temperature in Rayleigh-Bénard thermal turbulence from [31]; (cyan filled-o) experimental scaling exponents for temperature in heated turbulence with $130<R_{\lambda}<470$ from [16]. Two lines correspond to a variant of the SL model for passive scalar due to Leveque et al. [20] and due to He et al. [45]

It is interesting to note that Ruiz-Chavarria et al. [16] demonstrated experimentally that the model of hierarchy does not apply directly to the temperature structure functions. This drives Chen and Cao to develop bi-variate log-Poisson model [44]. He et al. [45] made a further improvement on a hierarchical model for joint moments of the passive scalar dissipation and the velocity dissipation, and calculated the scaling exponents of the passive scalar structure functions, which reads

$$
\begin{aligned}
z_{p}=3 & -\frac{p}{36}-2\left(\frac{3}{4}\right)^{p / 6}-\left(\frac{1}{2}\right)^{p / 2} \\
& +\gamma\left[1-\left(\frac{3}{4}\right)^{p / 6}-\left(\frac{1}{2}\right)^{p / 2}+\left(\frac{3}{4}\right)^{p / 6}\left(\frac{1}{2}\right)^{p / 2}\right] .
\end{aligned}
$$

This formula agrees with the experimental results (see Fig. 3). This work is a significant breakthrough in the framework of the SL hierarchical symmetry; it shows the necessity of introducing multiple hierarchies into the physical systems as the complexity rises. Future studies in this direction should clarify the nature of hierarchy as the principle of the selforganization.

\section{Hierarchical symmetry in magnetohydrodynamic turbulence}

Magnetohydrodynamic (MHD) is an important physical system with applications in many areas, including the motions of 
plasma in thermonuclear fusion reactor, the steel casting process in metallurgy industry, the power generation in propeller device, the electrolyte process in chemical industry, and the astrophysical applications of star's interior motion, solar wind, interstellar medium and molecular clouds, and so on. From a physical point of view, MHD of electrically conducting fluids involves more complexity because of the coupling to the electromagnetic force, and MHD turbulence displays both multi-scale behavior and the spatial anisotropy due to the magnetic field. Dynamical variables include both velocity $\boldsymbol{v}$ and magnetic field $\boldsymbol{B}$, and equations include both the Navier-Stokes equations for fluid and Maxwell's equation for electromagnetism. This complexity gives a particular fertile ground for one to appreciate the value of the SL hierarchical symmetry in modeling the macroscopic statistical behavior.

Traditional MHD turbulence theory adopted an Iroshnikov-Kraichnan (IK) view [46,47], which emphasizes its Alfvén wave nature. This theory assumes isotropy of the energy cascade in Fourier space, and predicts a unity scaling for the 4 th-order structure function $\left(\zeta_{4}=1\right)$. This view has received many criticisms, and it became quite clear [48] that small-scale eddies can not be free from the influence of the magnetic field [49]. A number of attempts have been made to apply the models of hydrodynamic turbulence such as the log-normal model, $\beta$-model, $p$-model and so on, to MHD turbulence, but until the appearance of the SL scaling law, the success had been very limited.

Grauer et al. [50] pioneered the use of the concept of SL hierarchical symmetry in the study of the MHD turbulence. Introducing the Elsässer variable $z^{ \pm}=\boldsymbol{v} \pm \boldsymbol{B}$ and using the two basic assumption of the SL model, namely the hierarchical symmetry and the most intermittent structure, they showed that the scaling of the structure functions is given by

$\zeta_{p}=p / 8+1-(1 / 2)^{p / 4}$.

This formula free of any adjustable parameter stems from the assumption that the most intense fluctuation structure is the current sheet with dimension two. This is an important breakthrough, as it shows that the original SL theory contains physical elements generalizable to multi-scale systems other than the hydrodynamic turbulence, and the SL hierarchical symmetry is more general. It was interesting that the above prediction found good agreement with the measured exponents by Burlaga [51] from solar wind. Grauer et al. [50] clearly stated that "the results were sufficiently encouraging to stimulate future numerical, experimental and observational efforts, in order to subject the adaption of the She-Leveque theory to a more stringent test". Soon, the same scaling exponent formula was derived by Politano et al. [52].

Further major progress was achieved owing to the development of high-resolution 3-D incompressible MHD simulations. Müller and Biskamp [53,54] reported the direct observation of sheet-like structures, and at the same time noticed that the energy spectrum is close to Kolmogorov's prediction (which predicts $\zeta_{3}=1$ ), rather than the IK theory (which predicts $\zeta_{4}=1$ ). They then modified the earlier Grauer analysis [50] and predicted a new scaling formula for the scaling:

$\zeta_{p}=p / 9+1-(1 / 3)^{p / 3}$.

Here again, the 2D sheetlike dissipative structures are assumed. This formula predicts, in particular, the value of the energy spectrum, $\zeta_{2} \simeq 0.7$, which is more consistent with the solar wind data. The scaling (11) has become one of the most popular formula in MHD turbulence.

In 2003, Müller and Biskamp [55] made a summary about the development of the phenomenological MHD turbulence models and the comparison with results of experimental measurements and direct numerical simulations. It is clear that the SL hierarchical symmetry has become the foundational concepts in those successful models of the two-point structure functions. They described also an excellent story of how to make a good combined effort from both phenomenology and data of numerical simulation to increase the physical understanding of the MHD turbulence. This is illustrated with a careful analysis of the anisotropic effect of the magnetic field in both decaying and forced MHD turbulence without and with a mean magnetic field [56]. This anisotropy is casted into a one-parameter family of scaling formula

$\zeta_{p}=p / g^{2}+1-(1 / g)^{p / g}$.

This expression was found in very good agreement with the results of the numerical simulations at different conditions [57]; hence, an interesting "g-class" of multi-scale phenomena are discovered.

The above analysis focuses on the Elsässer field $\left(z^{ \pm}\right)$, the other two fields (velocity $\boldsymbol{v}$ and magnetic field $\boldsymbol{B}$ ) are equally important physically. Cho et al. [58] carried out a detailed analysis on a 3D numerical simulations of driven incompressible MHD turbulence with a moderately strong external magnetic field, and this work has attracted a great deal of attention owing to its abundant statistical measures for MHD turbulence. A local frame of reference defined by the magnetic field is added in addition to the global Eulerian frame of reference. They [58] showed that the velocity structure functions in the plane perpendicular to the local mean magnetic field is quantitatively described by the original SheLeveque formula (1), while those parallel to the field were 1.5 times higher. This is consistent with the idea that eddies are stretched along the directions of the local magnetic field, and once this enhanced effect is taken into account, the similarity between hydrodynamic and MHD turbulence reappears. 
It is interesting $[59,60]$ to note the scaling exponents in the local frame for the magnetic field $\boldsymbol{B}, \zeta_{p}^{B, \text { local }}$, followed the Müller formula (11) at small $p$, but tended to level off at large $p$. This means that magnetic field is more intermittent than velocity. On the other hand, the scaling exponents calculated in the global frame is in good agreement with the Müller formula (11). In fact, in both local and global measurements, a rule is found to always hold, namely, the exponents for the Elsässer variables $z^{ \pm}$lie between those for the velocity and magnetic fields:

$\zeta_{p}^{B}<\zeta_{p}^{z}<\zeta_{p}^{v}$

These results can be understood in the framework of the SL hierarchical symmetry. First, we conclude that the most intense dissipative structures for magnetic field contain discontinuous jumps, which is more singular than the other two fields. In addition, both the measurement in the global frame and the Elsässer field contain a mixture of fluctuations, all of which satisfy the SL hierarchical symmetry. This explains the reason why the Müller formula (11) gives an overall good description.

MHD turbulence, on one hand, has more complex properties than hydrodynamic turbulence. The studies discussed above show that the SL hierarchical symmetry has been instrumental in deriving qualitative and quantitative understanding of the statistical properties of the MHD turbulence. On the other hand, the needs for understanding more complex physics than in the hydrodynamical turbulence offer a ground for the further development of the SL model. Some classical results following SL hierarchical symmetry in MHD turbulence have been shown in (Fig. 4).

\section{Hierarchical symmetry in astrophysics}

Motions of fluids in astrophysical environments typically extend to a wide range of scales, and intermittency seems to be their essential features. Knowledge about heating, momentum transfer, interaction with cosmic rays, radio waves and other processes require a good understanding of the statistical dynamics of fluids. The existence of intermittency violates the self-similarity of motions across scales, and it is an important question how to extrapolate the properties of a fluid obtained computationally with relatively low resolution to the actual astrophysical situations. Furthermore, the astrophysical environment provides a unique laboratory in which theoretical concepts can be tested with observations and large-scale computations. As we will show below, the concept of the hierarchy and the most intermittent structure is found to be instrumental in explaining observational data at astronomical scales, and in providing theoretical foundation for future theory of large-scale structure formation in the universe and of star formations.

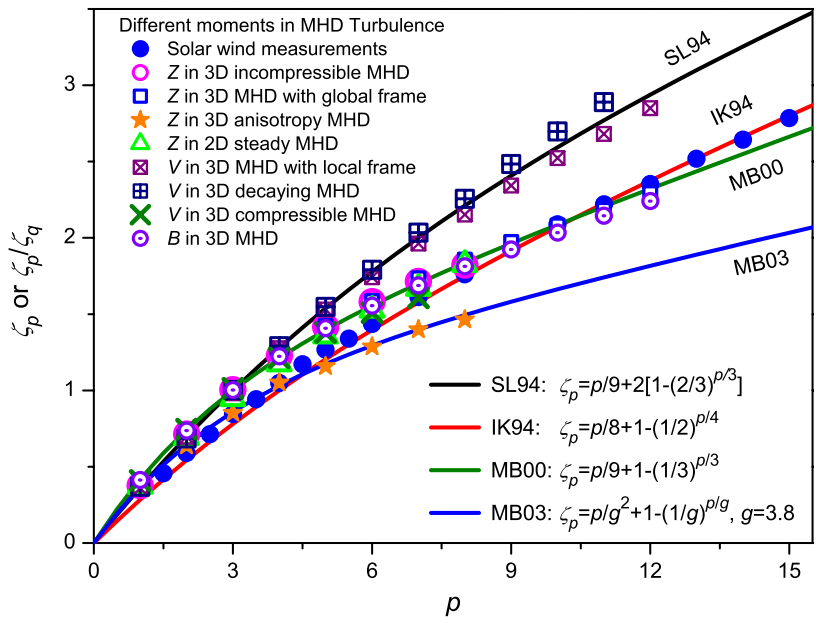

Fig. 4 Verification of the SL hierarchical symmetry in MHD turbulence: $(\bullet)$ observational results from solar wind from [50]; $(\bigcirc)$ computational results of 3D MHD turbulence from [53]; ( $\square$ ) computational results of 3D MHD turbulence in the global frame from [61]; $(\star)$ computational results of 3D anisotropic MHD turbulence from [56]; $(\triangle)$ computational results of 2D stationary MHD turbulence from [62]; $(\triangle)$ computational results of 3D MHD turbulence in a local frame from [58]; $(\boxplus)$ computational results of 3D decaying MHD turbulence from [61]; ( $)$ computational results of 3D compressible MHD turbulence from [63]; $\odot$ ) computational results of scaling of magnetic field of 3D decaying MHD turbulence from [61]). Four lines correspond to four models, all satisfying the SL hierarchical symmetry: SL94 [1], IK94 [50], MB00 [53], and MB03 [55] respectively

\subsection{Intermittency of cosmic baryon fluid}

The motion of cosmic baryon fluid is important to the largescale structure formation of the universe. In the intergalactic medium, the cosmic baryon fluid is compressible and dominated by the gravitation of dark matter, and the nonlinear stage of its evolution is crucial to the formation of luminous objects. The dynamics of its velocity fields are governed by the Burgers equation driven by the random force of the gravity of dark matter. The state of cosmic baryon fluid consists of strong sheet-like shock structures as the main dissipative structures which display strong intermittency effects and non-Gaussian behavior [64].

He et al. [65] conducted detailed numerical studies of this turbulence field, and concluded that velocity fluctuations in the scale larger than the Jeans length "can be extremely well described by She-Leveque's universal scaling formula". Its scaling is in excellent agreement with a modified SL scaling formula with $C=1$ and $\beta^{3}=1 / 3$. Here again, a simple argument that assumes sheet-like structures as the dominant emergent structure allows the authors to determine quantitatively the scaling exponents. Liu and Fang [66] conducted further studies on the mass density field of the cosmic baryon fluid, and found that its scaling is also "extremely well described by the SL hierarchy", and that "all the predictions given by the SL model, including the hierarchical 
relation, the order dependence of the intermittency exponent, the moments, and the scale-scale correlation, are in good agreement with the results given by hydrodynamic simulations of the standard cold dark matter model". The measured parameter $\beta^{\prime}=0.69 \pm 0.02$ happens to be in perfect agreement with the predicted parameter $\beta=(1 / 3)^{1 / 3} \approx$ 0.69 , which illustrates the power of the theory.

More recently, Lu et al. [67] studied the non-Gaussian nature of the mass density field of neutral hydrogen fluid, which can be studied using Ly $\alpha$ absorption spectrum. Using high resolution and high signal over noise data of quasars Ly $\alpha$ absorption spectrum, they show that all the non-Gaussian features predicted by the SL hierarchy, including the hierarchical relation, the intermittent exponents, the ratios of different moments, and the scale-scale correlation, are consistent with observed data [67]. In addition, careful comparison shows that the log-normal model predicts too strong non-Gaussianity at high orders, in sharp contrast with the SL model.

The first interesting message delivered from the above study is that turbulence of cosmic baryon fluid behaves similarly with respect to the form of the self-organization, i.e., the hierarchical symmetry. This is intriguing, since the SL model seems to give both the hydrodynamic turbulence and the compressible cosmic baryon fluid a satisfactory description of the scaling. Not only several theoretical predictions agree with the experimental and numerical findings, the variation of model parameters yields also insight about the physical properties of the system. For instance, it provides an excellent example that the intermittent behavior of the velocity fluctuations can be independent of the details of the dissipative processes. The fact that the parameter $\beta$ decreases with the decrease of redshift indicates that the field is more "homogeneous" with respect to the hierarchy at earlier time of the expansion, but becomes more "intermittent" at later time [66]. It implies also that the characteristic emergent structures of the density fluctuations are more singular at later stage of the expansion.

Generally speaking, using the SL hierarchical symmetry theory, the authors mentioned above are able to conclude that the clustering of the cosmic baryon fluid in gravitational field of an expanding universe has to be self-similarly hierarchical in the scale free range. So, the hierarchical symmetry seems to be a general form of the self-organization of nonlinear multi-scale systems, which implies the existence of a welldefined statistical ensemble and will play an important role in the future development of quantitative theory. Since above the Jeans length is the scale for the formation of the luminous objects, the characterization of the scaling behavior is important to the dynamical and thermodynamical evolution of structure formation. In particular, the redshift dependence of the scaling behavior would be useful for understanding the evolution of cosmic clustering.

\subsection{Supersonic turbulence for star formation}

Supersonic turbulence is ubiquitous in the interstellar medium and plays an important role in star formation, which occurs primarily inside cold interstellar clouds and is believed to be controlled by driven supersonic magnetohydrodynamic turbulence. The process of star formation begins with supersonic turbulent motion of the interstellar gas which develops shocks that interact with each other and with the turbulent flow, and this results in the emergence of rather complicated density structures. This process of turbulent fragmentation leads to creation of complicated density structures and sets the initial conditions for the collapse of small Jeans-unstable cores, which then leads to star formation. Thus, the understanding of supersonic turbulence is crucial for a satisfactory theory of star formation.

Boldyrev [68,74] suggested a Kolmogorov-Burgers model for supersonic turbulence which contains sheetlike shocks as the primary dissipative structure. He followed closely the ideas of the SL model and predicted a new scaling formula for the velocity structure functions:

$\zeta_{p} / \zeta_{3}=p / 9+1-(1 / 3)^{p / 3}$.

This formula happens to be the same as that obtained by Müller and Biskamp [53] for incompressible MHD turbulence, but the two systems are widely different physically and one finds an evidence of a general university class. A specific outcome of Boldyrev's theory is the prediction of the velocity power spectrum in the inertial range to be $E_{k} \sim k^{-1.74}$, a result known to be Larson's law from earlier observation. This is a remarkable outcome, because it links the most singular turbulent structures (sheet) with the exponent of the turbulent energy spectrum, which are previously unrelated. It is made possible only under the phenomenology of the SL hierarchical symmetry. The theoretically predicted exponents of the first four structure functions, namely $0.42,0.74$, 1.21 , and 1.40, are in excellent agreement with the results of a large-scale simulation, which are $0.42,0.74,1.20$, and 1.38, respectively (see Fig. 5).

Padoan et al. [69] studied the statistical property of the integrated intensity images of Taurus and Perseus molecular cloud complexes. They demonstrated that the moments satisfy the SL hierarchical symmetry, and the normalized scaling exponents of the structure functions are very close to those of the velocity in supersonic turbulence. This is taken as a direct evidence that the density fluctuation fields of the molecular cloud follows a hierarchical structure of the velocity fluctuations in supersonic turbulence and that turbulence plays an important role in the fragmentation of cold interstellar clouds. It motivated the authors [75] to propose a unified theory of the velocity scaling in compressible and superAlfvenic turbulence for a wide range of sonic Mach number, $M_{S}$. They found that under the She-Leveque formalism, the 


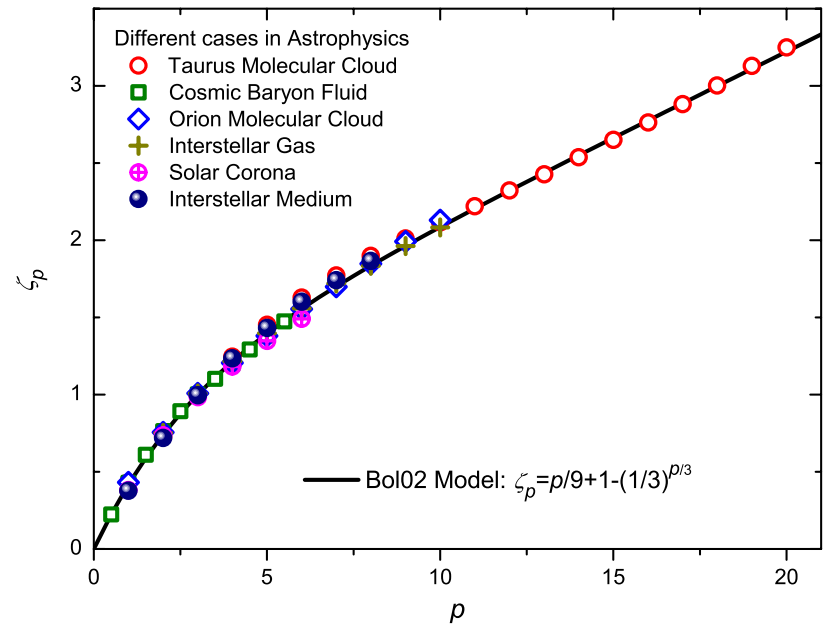

Fig. 5 Confirmation of the SL hierarchical symmetry in several astrophysical applications: (o) observational results for Tuarus molecular cloud images from [69]; ( $\square$ ) computational results of velocity scaling of cosmic baryon fluid from [65]; $(\diamond)$ computational results for Orion molecular cloud from [70]; (+) computational results for interstellar medium from [71]; $(\oplus)$ observational results for solar corona images from [72]; (ball) computational results for interstellar medium from [73]. The solid line correspond to modified SL model by Boldyrev [74]

only model parameter, the Hausdorff dimension of the most intense dissipative structures, can be linked to the sonic Mach number. The latter can be inferred from observations, and the derived Hausdorff dimension appears to be very reasonable and is believed to be an important property characterizing the interstellar clouds. Here, as a phenomenological theory, the SL model is able to yield a meaningful relation between two important physical parameters.

Very recently, Pan et al. [76] conducted a detailed study of the dissipative structure in numerically simulated supersonic turbulence at a Mach number 6. In addition to the evidence showing the applicability of the SL model to supersonic turbulence, they verified that the SL parameters characterizing the most intense dissipative structures carry a precise physical meaning. They developed a general method to directly measure those parameters, and the application of the method provides a validity test of the physical interpretation of the model. The measured SL parameters are in good agreement with the ones used for describing the scaling. The successful application of the SL model to supersonic turbulence has been made extremely convincing [76].

Several other numerical studies have delivered the same message, which have been shown in Fig. 5. Using large-scale high-resolution simulations, Avillez and Breitschwerdt [71] studied the generation and dissipation of interstellar turbulence, and showed that the scalings of the structure functions are consistent with the SL model of supersonic turbulence, where energy is dissipated mainly through shocks. Kissmann et al. [73] also performed numerical simulations for turbulence in the interstellar medium, and found that the dissipative structures quantitatively follow the SL model. Kritsuk and Norman [77] have studied the scaling relations in more complex multiphase interstellar medium with turbulence induced by thermal instability. Due to phase transition and radiative cooling, such multiphase turbulence tends to be more intermittent, and the Hausdorff dimension of the most singular dissipative structures can be as high as 2.3. The SL model needs to be generalized in the multiphase medium, where one may find interesting new consequences on the dynamics behavior of molecular clouds and on star formation.

In summary, turbulence is self-organized around the most intermittent structure, and the form of the self-organization is the hierarchical symmetry. Supersonic turbulence provides abundant evidence to support this vision. Hierarchical clustering is a common feature of all star-forming regions [78], and the hierarchical structures of turbulent fluctuations may be its origin. However, the development is far from being complete yet, but it appears to be promising for a selfconsistent treatment of turbulence in the complex scenarios encountered in star-forming clouds.

\section{Hierarchical symmetry in other multi-scale systems}

The SL hierarchical symmetry addresses principally the issue of scaling which is one of the most sensitive measures for multi-scale systems. The theory is a specific example of multifractal models á la Frisch and Parisi [79], but is significantly different from other specific multifractal models such as $\beta$-model [80], $p$-model [81], random- $\beta$ model [82], in that the SL model is developed around a symmetry and a most significant structure of the field, rather than an abstract multiplicative cascade process. The connection to physical structures is the principal reason why the SL model is popular around astrophysicists, and why the SL model is also applicable to a variety of other systems where the cascade picture is not so obvious.

A variety of nonlinear systems with multi-scale fluctuations have been found to exhibit the hierarchical symmetry. Spatio-temporally chaotic spiral patterns [83], the diffusionlimited aggregates [84], a fluctuating luminosity field of nature images $[85,86]$, a nucleotide density variation along DNA sequences [87-89], climate variations [90,91], etc. is a short list from several dozen published examples. We focus the discussion on a subset of those mentioned above.

Throughout the examples, we will illustrate a point, namely, using the hierarchical symmetry, the global property of a multi-scale fluctuating field can be extracted and synthesized, and the SL parameters often, if not always, give a quantitative description of the global statistical property of the system. 


\subsection{Spatiotemporal chaos}

Spatiotemporally developed chaotic spiral patterns are intriguing structures in macroscopic systems driven far from thermodynamic equilibrium. The Belousov-Zhabotinsky (BZ) chemical reaction-diffusion system [92] is found to display similar patterns as those found in biological systems such as cardiac muscle tissue $[93,94]$. Experimental studies reveal a series of transitions from one type of spiral turbulence to another, and between spiral turbulence and ordered spiral waves [83]. But the characterization of spatiotemporally chaotic states had not been satisfactory due to the large numbers of degrees of freedom. Liu et al. [6] investigated this problem based on the SL theory.

The main idea is that any statistically sustained state of spatiotemporal patterns/chaos involves fluctuations across scales which are generally describable in terms of the hierarchical symmetry. The application of the hierarchical symmetry analysis requires a good definition of statistical moments or structure functions. Liu et al. [83] applied the method of so-called $\beta$-test and $\gamma$-test [5] using the following intermediate variables,

$G(x, y)=\sqrt{\left(\frac{\partial f}{\partial x}\right)^{2}+\left(\frac{\partial f}{\partial y}\right)^{2}}$,

where $f(x, y)$ is the chemical concentration in the BZ reaction experiment. Then using a multi-scale random variable,

$\epsilon_{\ell}=\frac{1}{l^{2}} \int_{l}^{x+l} \int_{y}^{y+l} G(x, y) \mathrm{d} x \mathrm{~d} y$,

one can define the structure function, $S_{p}(l)=\left\langle\epsilon_{\ell}^{p}\right\rangle$. Detailed calculation using experimental data and that of the numerical simulation of the two-dimensional complex GinzbergLandau equation $[6,7]$ (which yields similar spiral patterns) showed that the hierarchical symmetry is very well observed. Furthermore, the parameter $\beta$ gives a quantitative description of the degree of heterogeneity of the patterns. For large $\beta$ close to one, the system appears to be orderly homogeneous (pure spirals) For smaller $\beta$, the system noticeably contains a mixture of ordered spirals and disordered waves, and thus appears to be heterogeneous. The smallest $\beta$ is observed to be associated with the appearance of overwhelmingly disordered and intermittent state which can be characterized as fully developed spatiotemporal chaos.

Spatiotemporal chaos such as the spiral turbulence does not display a substantial cascade as hydrodynamic turbulence. Nevertheless, nonlinear interaction spreads excitations across a certain range of scales and fluctuations spread over a certain range of intensities. The successful application of the SL theory suggests that the hierarchical symmetry is a principle which is valid for general nonlinear systems with spatiotemporal disordered across scales. This is the reason why the SL parameter $\beta$ gives a sound description of the state of chaos.

\subsection{Natural images}

Natural monochrome images which possess a very rich set of geometrical structures need to be described in terms of multiscaling relations. The SL hierarchical symmetry is again proved to be a principle that is found very useful for characterizing the statistical property of natural images. Turiel et al. [85] pioneered a study in this direction. A random variable representing the local fluctuation of luminosity $\epsilon_{r}$, called local edge variance, is shown to resemble the local energy dissipation in turbulent flows, and the moments satisfying the scaling relation $\left\langle\epsilon_{r}^{p}\right\rangle \propto r^{\tau_{p}}$ are shown to obey the SL hierarchical symmetry, which yields a scaling formula of the SL type,

$\rho_{p, q}=\frac{1-\beta^{p}-(1-\beta) p}{1-\beta^{q}-(1-\beta) q}$.

This formalism enables the above authors to achieve a decomposition of pixels of the image into fractal components [95] and to gave a precise measure of their informational relevance [86]. Recently, applying the method to the analysis of satellite meteorological images, Grazzini et al. [96] found that the temperature distribution and a synthetic graylevel distribution are both consistent with the prediction of the hierarchical symmetry. Furthermore, a most singular manifold (MSM) analogous to the most intermittent structure in the original SL formulation is defined and used to characterize the image. The fractal dimension and the singularity exponent of the MSM are found to determine all the multifractal properties. In this application, the SL theory becomes a standard tool in the so-called multi-scale methodology (see Fig. 6).

\subsection{DNA sequences}

DNA is a one-dimensional array of texts of nucleotides, but contains the most of, if not all, information about the life system. It is intriguing to develop statistical tools to capture the information at a global level. The SL hierarchical symmetry has become such a tool of multi-scale methodology.

A locally averaged density over a window of size $L$ is defined as,

$\rho_{L}^{I}=\frac{1}{L} \sum_{i=1}^{L} \delta_{u_{i}}^{I}$,

where $I$ denotes the nucleotide, $\mathrm{I}=\mathrm{A}, \mathrm{T}, \mathrm{G}$ and $\mathrm{C} . \rho_{L}^{I}$ typically displays multi-scale fluctuations with statistical property dependent on $L$. Thus, the structure functions, 


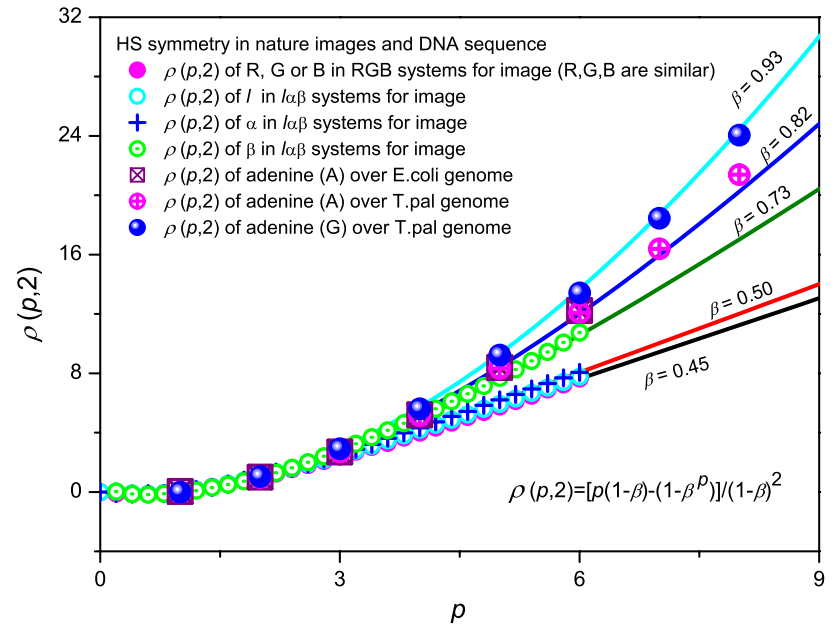

Fig. 6 Confirmation of the SL hierarchical symmetry in applications to natural images and DNA sequences: $(\bullet)$ computed ESS exponents $\rho(p, 2)$ of $\mathrm{R}, \mathrm{G}$ or B in RGB image systems from [86]; $(\circ,+$ and $\odot)$ computed ESS exponents $\rho(p, 2)$ of $l, \alpha$ and $\beta$ in $l \alpha \beta$ image systems from [86]; $(\nabla)$ computed ESS exponents $\rho(p, 2)$ of A from E.coli DNA sequences from [87]; ( $\oplus$ and ball) computed ESS exponents $\rho(p, 2)$ of $\mathrm{A}$ and $\mathrm{G}$ from T. pal DNA sequences from [88]. Five curves correspond to modified SL scaling due to Turiel et al. [85] and Ouyang et al. [88]

$S_{p}^{I}(L)=\left\langle\left(\rho_{L}^{I}\right)^{p}\right\rangle$, are introduced to describe this multi-scale variation. Using the sequence of $E$-coli complete genome, Wang et al. [87] reported the first evidence that the SL hierarchical symmetry holds for all I; furthermore, based on the measurement of the SL parameters, they showed that the $\mathrm{G}, \mathrm{C}$ density distributions are less intermittent than that of A, T, which is in accordance with their chemical property (three chemical bonds versus two). Later, Ouyang et al. [88] extended the analysis to DNA sequences of all categories (from bacterial to human) and showed that the relative scaling of $S_{p}^{I}(L)$ for all $I$ is accurately described by a variant of the SL model:

$\zeta_{p} / \zeta_{2}=\frac{(1-\beta) p-\left(1-\beta^{p}\right)}{(1-\beta)^{2}}$,

and the variation of $\beta$ describes the evolution category of the genome and measures its organizational complexity. Compared to earlier statistical analysis using the energy spectrum, entropy or frequency distribution of k-tuples, the SL analysis reveals an organizational order of the genome at a higher level, which has been shown in Fig. 6.

Furthermore, Ouyang et al. [89] used the SL analysis as a tool to study the origin of the intermittent fluctuations in $\rho_{L}^{I}$, and identified the lateral transfer of genomic material as the main source of the abnormal base composition. Remarkably, after a detailed analysis carried out for over one hundred bacteria, archaea, virus, yeast, and human genome sequences, Ouyang et al. [89] showed that the SL parameter $\beta$ is a characteristic measure of the genome, which reveals an intriguing link to evolutionary events of genetic material transfer.

\subsection{Human heart variability}

Recent studies [97] indicated that human heart variability (HRV) exhibits a very high degree of complexity, and the degree of the complexity varies between the healthy and the pathological state. It is thus important to develop measures that are capable for characterizing the multi-scale complexity of HRV. Again, the SL hierarchical symmetry finds its application for develop a method of analysis.

Ching et al. [98] reported substantial evidence for the existence of the SL hierarchical symmetry in both healthy and diseased HRV, and this immediately allows the authors to model the multifractal HRV in terms of only two parameters, $\mathrm{C}$ and $\beta$, and to go beyond the general multifractal framework for more quantification of the HRV analysis. In particular, Ching et al. [98] discovered a relation between the two parameters,

$C \approx 0.2 \beta / \ln (1 / \beta)$.

This empirical law seems to be universal and is capable of describing both healthy and congestive heart failure data. Moreover, the diseased HRV shows noticeable tendency of divergent increase of the value of $\mathrm{C}$, implying the potential in diagnosis of congestive heart failure based on the characteristics derived from the SL hierarchy.

In summary, the SL hierarchical symmetry allows one to model the multifractality of HRV and make conjecture to the heart beat dynamics responsible for the multifractal scaling, which achieves an understanding beyond the traditional phenomenological description of multifractal HRV.

\section{Future development: self-organization and turbulence ensemble}

\subsection{Principle of self-organization from chaos to turbulence}

Throughout the examples we discussed in this article, we would like to argue that self-organization is a key concept. Often, it appears so abstract to any practical use, but a deep thinking tells us that it is fundamental to the success of the SL description.

A classical example that illustrates the development of the ideas of self-organization is the Rayleigh-Bénard thermal convection. At small temperature differences between two horizontal plates or at small Rayleigh numbers, the fluid is at rest and the thermal motion is of Brownian type, which drives a conductive heat flux. At this stage, there is no self-organized macroscopic motion. As the temperature difference increases or at higher Rayleigh numbers, convective motions occur from instabilities and nonlinear saturation mechanism. This is the first set of macroscopic self-organization which is described mathematically by a set of amplitude equations 
Table 1 Classical HS scaling models

\begin{tabular}{|c|c|c|}
\hline Year & Authors & Formula \\
\hline 1994 & She and Leveque [1] & $\zeta_{p}=\frac{p}{9}+2\left[1-\left(\frac{2}{3}\right)^{p / 3}\right]$ \\
\hline 1995 & She and Waymire [3] & $\zeta p=\gamma p+C\left(1-\beta^{p}\right)$ \\
\hline 1994 & Dubrulle [2] & $\frac{\zeta p}{\zeta_{3}}=(1-\Delta) \frac{p}{3}+\frac{\Delta}{1-\beta}\left(1-\beta^{p / 3}\right)$ \\
\hline 1994 & Grauer et al. [50] & $\zeta_{p}=\frac{p}{8}+1-\left(\frac{1}{2}\right)^{p / 4}$ \\
\hline 1995 & Politano and Pouquet [52] & \\
\hline 1995 & Cao and Chen [34] & $\zeta_{p}=\frac{9}{2}\left[1-\left(\frac{7}{9}\right)^{p / 3}\right]$ \\
\hline 1998 & He et al. [45] & $\begin{aligned} z_{p}= & 3-\frac{p}{36}-2\left(\frac{3}{4}\right)^{p / 6}-\left(\frac{1}{2}\right)^{p / 2} \\
& +\gamma\left[1-\left(\frac{3}{4}\right)^{p / 6}-\left(\frac{1}{2}\right)^{p / 2}+\left(\frac{3}{4}\right)^{p / 6}\left(\frac{1}{2}\right)^{p / 2}\right]\end{aligned}$ \\
\hline 1998 & Turiel et al. [85] & $\rho_{p, 2}=\frac{(1-\beta) p-\left(1-\beta^{p}\right)}{(1)^{2}}$ \\
\hline 2004 & Ouyang et al. [88] & $(1-\beta)^{2}$ \\
\hline 1999 & Leveque et al. [20] & $\zeta_{p}=\frac{p}{9}+\frac{10}{9}\left[1-\left(\frac{2}{5}\right)^{p}\right]$ \\
\hline 2000 & Müller and Biskamp [53] & $\zeta_{p}=\frac{p}{9}+1-\left(\frac{1}{3}\right)^{p / 3}$ \\
\hline 2002 & Boldyrev [68] & \\
\hline 2001 & Biskamp et al. [99] & $\frac{\zeta_{p}}{\zeta_{3}}=\frac{4}{15} p+\frac{1}{3}\left[1-\left(\frac{2}{5}\right)^{p / 3}\right]$ \\
\hline 2003 & Müller and Biskamp [56] & $\zeta_{p}=\frac{p}{g^{2}}+1-\left(\frac{1}{g}\right)^{p / g}$ \\
\hline 2004 & Haugen et al. [100] & $\frac{\zeta_{p}}{\zeta_{3}}=\frac{p}{9}+C\left[1-\left(1-\frac{2}{3 C}\right)^{p / 3}\right]$ \\
\hline 2008 & Liu and Fang [66] & $K_{p}=-\gamma \frac{p\left(1-\beta^{2}\right)-\left(1-\beta^{2 p}\right)}{1-\beta}$ \\
\hline 2009 & Lu et al. [67] & $\xi_{p}=-\alpha p-\gamma\left[p-\left(1-\beta^{p}\right) /(1-\beta)\right]$ \\
\hline
\end{tabular}

arising from the asymptotic analysis on the continuum field equations.

Soon, as the Rayleigh number further increases, the convective cells break and more fluctuations occur, and the motion becomes temporally chaotic and spatially disordered. As the spatial disorder increases, the asymptotic amplitude equations cease to be valid, and the thermal convection as a dynamical system enters to a phase space of large dimension. In other words, the macroscopic self-organization needs to find a new set of variables that are different from the ones used in the amplitude equations.

Note that the amplitude equations or the dynamical system equations for such system as thermal convection are the mathematical form of the self-organization. The role of the self-organization, at this stage, is hidden in the derivation of the amplitude equations, evidenced by the fact that the functional form of the amplitude equations are surprisingly universal and valid for a surprisingly large domain of the parameters, far from the critical point. The principle of this self-organization can be better understood in terms of the preservation of symmetry, which played a central role in the derivation of equations.

The development of turbulence in the Rayleigh-Bénard convection increases drastically the amount of disorder. When following the traditional dynamical system approach, one encounters a large number of bifurcations, each of which is associated with a symmetry-breaking. The continuing attempts using macroscopic variables in the dynamical system theory meet huge obstacles, and new ideas about the self-organization are needed.

Turbulent Rayleigh-Bénard convection have two remarkable features. The first is the appearance of multi-scale fluctuations which are neither regular nor random. The second is the appearance of thin plumes which has attracted much attention, but has offered little in quantitative understanding of the system. Until now, these two aspects have not been satisfactorily reconciled and incorporated into a single theoretical framework.

Note that the existence of scaling for various structure functions (absolute or relative) is, by itself, a proof for the 
self organization, because the scaling dictates the way how moments change with scales and how small-scale eddies are related to large-scale ones. However, the SL hierarchical symmetry enriches the content of the self-organization of multi-scale systems in a specific way; it reveals the fact that various fluctuation intensities are "hierarchically"-organized so that high intensities become increasingly more coherent, and this organization follows a specific rule. This is a new form of the self-organization principle, which assembles the whole set of multi-scale fluctuations into a single ensemble, which will be referred to as the turbulence ensemble and will be discussed in detail in the next subsection.

What is the center of the self-organization as turbulence is fully developed? According to the SL hierarchical symmetry, the only characteristic structure of a fully turbulent system is the most intermittent structure, under certain physical conditions to be specified. No other weaker fluctuation structures are special among themselves. This is a statement of symmetry, and is a statement about the principle of the selforganization. All fluctuations can be thought to be linked, with respect to the statistical ensemble, like "buckles" in a "chain"; all "buckles" are inseparable parts of the system, but only the one at the end of the "chain", the most intermittent structures, play a special role. The SL hierarchical symmetry is the "chain", and the center of the chain is the most intermittent structure, which is plume in the Rayleigh-Bénard convection, filament in isotropic incompressible turbulence, shock-sheet in the interstellar medium, and current sheet in incompressible MHD, etc. Without the chain, the rare events play no special role; hence, the SL hierarchical symmetry is, in our view, the new form of the symmetry and of the self-organization!

\subsection{SL hierarchical symmetry and turbulence ensemble}

The central problem in the study of turbulence is always to find a set of equations that determine its macroscopic/statistical property. In order to find a suitable set of equations, a series of concepts need to be created, among which we postulate a "turbulence ensemble". This is, in our view, a crucial stage for determining the role of statistical average of hydrodynamic fluctuation variables, before developing a cohesive statistical description of turbulence.

The turbulence ensemble is the ensemble which contains the most, if not all, relevant fluctuations quantified with welldefined probabilities and by a restricted set of parameters. The existence of this ensemble heavily relies on the principle of the self-organization; the validity of the SL hierarchical symmetry provides it with a solid base. The existence of this ensemble is in no conflict with the fact that turbulence is in a non-equilibrium state with driven flux in both scale space (in terms of cascade) and physical space (when inhomogeneity is important) by nonlinear interaction. The presence of the flux implies that the turbulence ensemble is very different from the thermal equilibrium ensemble, but its existence suggests a well-defined set of probability distributions which can be determined with a limited number of parameters, and consequently a well-defined set of relations between the mean, variance, and other high-order moments.

The SL hierarchical symmetry, although waiting for further theoretical justification, stands as the simplest candidate of such ensemble. The successful application of the SL model implies that the turbulence ensemble may be described, to a good approximation, by a hierarchical similarity parameter $\beta$ and the singularity index of the most intermittent structure $\gamma$ (or the co-dimension $C$ ). In more realistic non-homogeneous turbulence involving non-trivial spatial transport, the existence of the turbulence ensemble would change the way to derive the closure equations. In particular, with well-defined relations between low-order and high-order moments owing to the hierarchical symmetry, a set of equations that closely mimic the Navier-Stokes dynamics may not be impossible, and traditional ad hoc phenomenological closure assumptions may be avoided.

The importance of the turbulence ensemble manifests in ways to unambiguously evaluate statistical averages and to establish dynamical balances (and new equations) for a set of statistical variables. The tremendous obstacle lied before the closure problems of turbulence seems to be the lack of a faith for the existence of the turbulence ensemble and the lack of a firm searching for its form. While extensive efforts have been devoted to the accurate measuring of statistical correlations and to the search for the form of turbulent structures, we are still far from being able to determine the set of key variables and correlations that are sufficient for describing the variations of the statistical mean quantities and/or large-scale dynamics. It has even become less clear which is the set of dynamical variables crucial to describing turbulent structures (see [101] for a recent attempt) and their dynamics. The concept of the turbulence ensemble and its principle of the self-organization indicate a need for rethinking about the question.

In fact, the theory of SL hierarchical symmetry and the future theory of the turbulence ensemble sends out a new message, that is, a quantitative description of the turbulence ensemble requires descriptions at several different levels. First, at a grand self organizational level is the hierarchical symmetry parameter $\beta$; then, at a sub organizational level is the multi-scale behavior of the most intermittent structures described in terms of the singularity index $\gamma$; and finally, at the geometrical level is the co-dimension parameter $C$ of the most intermittent structure. The recognition of this multi-level description is at the heart of the SL theory, which provides a new basis for the description of the turbulence ensemble, and for future turbulence models of engineering purpose. 
Theoretical concepts relating the SL-related turbulence ensemble to the resolution of the closure problem are being developed at the Peking University by our group and termed "structure ensemble dynamics" (SED) approach to turbulence closure. The SED approach consists in analyzing the spatial variations of various correlations functions by closely following the Navier-Stokes dynamics under the multi-scale constraint such as the hierarchical symmetry. We will communicate the finding in the near future.

\subsection{SL hierarchical symmetry analysis as a general multi-scale methodology}

As we have indicated above, the SL hierarchical symmetry is a general principle which supports the self-organization of multi-scale fluctuation systems; it is the topological constraint of the "chain" behind the whole set of two-point statistical correlations. When fluctuations develop across a range of scales and across a range of intensities, such a constraint is unavoidable if the whole ensemble must sustain with the most intermittent structures as the only characteristic structure in the ensemble. We have collected a fairly large set of examples for making a convincing point. There should exist more multi-scale fluctuation systems like the ones mentioned in this articles, which deserve a close look and examination regarding the validity of the SL hierarchical symmetry. We believe that the recognition of the SL hierarchical symmetry would enhance the level of their description, and the parameters should, in general, improve our understanding.

In this regard, we propose to develop the SL hierarchical symmetry analysis as part of the general multi-scale methodology. We stress that an important feature of the SL hierarchical symmetry is that it can be tested directly using the structure function data from either experiments and numerical simulations; hence the SL hierarchical symmetry analysis may become a standard tool in the multi-scale methodology for all multi-scale, multi-intensity phenomena. Another important feature is its direct link to the geometrical property of the multi-scale fluctuation systems. Once the SL hierarchical symmetry is confirmed through the $\beta$-test [5], the structure function data can be used to further determine the property of the most intermittent structures. This information is central to the whole multi-scale fluctuation system, and should be used for quantification of the global property. We speculate that it will become a standard measure for most multi-scale fluctuation systems.

Acknowledgements The authors wish to thank S.Y. Chen, C.B. Lee, J. Chen, J.J. Tao, Y.P. Shi, X.T. Shi, N. Hu, Y. Wu, X. Chen, J. Pei, K. Ding, Y.Z. Wang for helpful discussions.

Open Access This article is distributed under the terms of the Creative Commons Attribution Noncommercial License which permits any noncommercial use, distribution, and reproduction in any medium, provided the original author(s) and source are credited.

\section{References}

1. She, Z.S., Leveque, E.: Universal scaling laws in fully-developed turbulence. Phys. Rev. Lett. 72(3), 336-339 (1994)

2. Dubrulle, B.: Intermittency in fully-developed turbulence: logpoisson statistics and generalized scale covariance. Phys. Rev. Lett. 73(7), 959-962 (1994)

3. She, Z.S., Waymire, E.C.: Quantized energy cascade and logpoisson statistics in fully-developed turbulence. Phys. Rev. Lett. 74(2), 262-265 (1995)

4. Kolmogorov, A.N., Acad, C.R.: Local structure of turbulence in an incompressible fluid for very large Reynolds numbers. Dokl. Akad. Nauk. SSSR. 30, 301-305 (1941)

5. She, Z.S., Ren, K., Lewis, G.S., Swinney, H.L.: Scalings and structures in turbulent Couette-Taylor flow. Phys. Rev. E 64, 016308 (2001)

6. Liu, L., She, Z.S.: Hierarchical structure description of intermittent structures of turbulence. Fluid. Dyn. Res. 33(3), 261-286 (2003)

7. Liu, J., She, Z.S., Guo, H.Y., Li, L., Ouyang, Q.Y.: Hierarchical structure description of spatiotemporal chaos. Phys. Rev. E 70, 2036215 (2004)

8. Frisch, U.P., Sul, P.L., Nelkin, M.: A simple dynamical model of intermittent fully developed turbulence. J. Fluid Mech. 87(4), 719-736 (2006)

9. Sinai, Y.G.: Statistics of shocks in solutions of inviscid Burgers equation. Commun. Math. Phys. 148(3), 601-621 (1992)

10. She, Z.S., Aurell, E., Frisch, U.: The inviscid Burgers equation with initial data of Brownian type. Commun. Math. Phys. 148(3), 623-641 (1992)

11. Benzi, R., Ciliberto, S., Tripiccione, R., Baudet, C., Massaioli, F., Succi, S.: Extended self-similarity in turbulent flows. Phys. Rev. E 48(1), 29-32 (1993)

12. Kadanoff, L.P.: Imports and exports. J. Stat. Phys. 111(5-6), 13911396 (2003)

13. Sreenivasan, K.R., Antonia, R.A.: The phenomenology of smallscale turbulence. Annu. Rev. Fluid. Mech. 29, 435-472 (1997)

14. RuizChavarria, G., Baudet, C., Ciliberto, S.: Hierarchy of the energy-dissipation moments in fully-developed turbulence. Phys. Rev. Lett. 74(11), 1986-1989 (1995)

15. Chavarria, G.R., Baudet, C., Benzi, R., Ciliberto, S.: Hierarchy of the velocity structure functions in fully-developed turbulence. J. Phys. II France 5(4), 485-490 (1995)

16. RuizChavarria, G., Baudet, C., Ciliberto, S.: Scaling laws and dissipation scale of a passive scalar in fully developed turbulence. Phys. D 99(2-3), 369-380 (1996)

17. Camussi, R., Benzi, R.: Hierarchy of transverse structure functions. Phys. Fluids 9(2), 257-259 (1997)

18. Benzi, R., Ciliberto, S., Baudet, C., Chavarria, G.R.: On the scaling of 3-dimensional homogeneous and isotropic turbulence. Phys. D 80(4), 385-398 (1995)

19. Benzi, R., Biferale, L., Ciliberto, S., Struglia, M.V., Tripiccione, R.: Generalized scaling in fully developed turbulence. Phys. D 96(1-4), 162-181 (1996)

20. Leveque, E., Ruiz-Chavarria, G., Baudet, C., Ciliberto, S.: Scaling laws for the turbulent mixing of a passive scalar in the wake of a cylinder. Phys. Fluids 11(7), 1869-1879 (1999)

21. Zou, Z.P., Zhu, Y.J., Zhou, M.D., She, Z.S.: Hierarchical structures in a turbulent pipe flow. Fluid. Dyn. Res. 33(5-6), 493-508 (2003)

22. Jiang, X.Q., Gong, H., Liu, J.K., Zhou, M.D., She, Z.S.: Hierarchical structures in a turbulent free shear flow. J. Fluid Mech. 569, 259-286 (2006)

23. Baroud, C.N., Plapp, B.B., Swinney, H.L., She, Z.S.: Scaling in three-dimensional and quasi-two-dimensional rotating turbulent flows. Phys. Fluids 15(8), 2091-2104 (2003) 
24. Lewis, G.S., Swinney, H.L.: Velocity structure functions, scaling, and transitions in high-Reynolds-number Couette-Taylor flow. Phys. Rev. E 59(5), 5457-5467 (1999)

25. Heslot, F., Castaing, B., Libchaber, A.: Transitions to turbulence in helium gas. Phys. Rev. A 36(12), 5870-5873 (1987)

26. Sano, M., Wu, X.Z., Libchaber, A.: Turbulence in helium-gas free-convection. Phys. Rev. A 40(11), 6421-6430 (1989)

27. Ching, E.S.C., Kwok, C.Y.: Statistics of local tperature dissipation in high Rayleigh number convection. Phys. Rev. E 62(6), R7587R7590 (2000)

28. Ching, E.S.C.: Intermittency of tperature field in turbulent convection. Phys. Rev. E 61(1), R33-R36 (2000)

29. Ching, E.S.C., Leung, C.K., Qiu, X.L., Tong, P.: Intermittency of velocity fluctuations in turbulent thermal convection. Phys. Rev. E 68, 026307 (2003)

30. Ching, E.S.C.: Intermittency and scaling in turbulent convection. Acta. Mech. Sin. 19(5), 385-393 (2003)

31. Sun, C., Zhou, Q., Xia, K.Q.: Cascades of velocity and temperature fluctuations in buoyancy-driven thermal turbulence. Phys. Rev. Lett. 97(14), 144504 (2006)

32. Cao, N.Z., Chen, S.Y., She, Z.S.: Scalings and relative scalings in the Navier-Stokes turbulence. Phys. Rev. Lett. 76(20), 37113714 (1996)

33. Sreenivasan, K.R., Vainshtein, S.I., Bhiladvala, R., SanGil, I., Chen, S.Y., Cao, N.Z.: Asymmetry of velocity incrents in fully developed turbulence and the scaling of low-order moments. Phys. Rev. Lett. 77(8), 1488-1491 (1996)

34. Chen, S.Y., Cao, N.Z.: Inertial range scaling in turbulence. Phys. Rev. E 52(6), R5757-R5759 (1995)

35. Grossmann, S., Lohse, D., Reeh, A.: Different intermittency for longitudinal and transversal turbulent fluctuations. Phys. Fluids 9(12), 3817-3825 (1997)

36. Fisher, R.T., Kadanoff, L.P., Lamb, D.Q., Dubey, A., Plewa, T., Calder, A., Cattaneo, F., Constantin, P., Foster, I., Papka, M.E., Abarzhi, S.I., Asida, S.M., Rich, P.M., Glendening, C.C., Antypas, K., Sheeler, D.J., Reid, L.B., Gallagher, B., Needham, S.G.: Terascale turbulence computation using the FLASH3 application framework on the IBM Blue Gene/L syst. IBM J. Res. Dev. 52(1-2), 127-136 (2008)

37. Boratav, O.N., Pelz, R.B.: Structures and structure functions in the inertial range of turbulence. Phys. Fluids 9(5), 1400-1415 (1997)

38. Boratav, O.N.: On recent intermittency models of turbulence. Phys. Fluids 9(5), 1206-1208 (1997)

39. Kida, S.: 3-dimensional periodic flows with high-symmetry. J. Phys. Soc. Jpn. 54(6), 2132-2136 (1985)

40. Haugen, N.E.L., Brandenburg, A.: Inertial range scaling in numerical turbulence with hyperviscosity. Phys. Rev. E 70, 026405 (2004)

41. Pumir, A.: A numerical study of the mixing of a passive scalar in three dimensions in the presence of a mean gradient. Phys. Fluids 6(6), 2118-2132 (1994)

42. Chen, S.Y., Cao, N.Z.: Anomalous scaling and structure instability in three-dimensional passive scalar turbulence. Phys. Rev. Lett. 78(18), 3459-3462 (1997)

43. Watanabe, T., Gotoh, T.: Statistics of a passive scalar in homogeneous turbulence. New J. Phys. 6(1), 40 (2004)

44. Cao, N.Z., Chen, S.Y.: An intermittency model for passive-scalar turbulence. Phys. Fluids 9(5), 1203-1205 (1997)

45. He, G.W., Chen, S.Y., Doolen, G.: Hierarchy of structure functions for passive scalars advected by turbulent flows. Phys. Lett. A 246(1-2), 135-138 (1998)

46. Iroshnikov, P.: Turbulence of a conducting fluid in a strong magnetic field. Astron. Zh. 40, 742 (1963)

47. Kraichnan, R.: Inertial-range spectrum of hydromagnetic turbulence. Phys. Fluids 8, 1385 (1965)
48. Sridhar, H., Goldreich, P.: Toward a theory of interstellar turbulence I. Weak Alfvenic turbulence. Astrophy. J. 432, 612 (1994)

49. Goldreich, P., Sridhar, H.: Toward a theory of interstellar turbulence. 2: Strong alfvenic turbulence. Astrophy. J. 438, 763 (1995)

50. Grauer, R., Krug, J., Marliani, C.: Scaling of high-order structure functions in magnetohydrodynamic turbulence. Phys. Lett. A 195(5-6), 335-338 (1994)

51. Burlaga, L.F.: Intermittent turbulence in the solar wind. J. Geophys. Res. 96(A4), 5847-5851 (1991)

52. Politano, H., Pouquet, A.: Model of intermittency in magnetohydrodynamic turbulence. Phys. Rev. E 52(1), 636-641 (1995)

53. Müller, W.C., Biskamp, D.: Scaling properties of three-dimensional magnetohydrodynamic turbulence. Phys. Rev. Lett. 84(3), 475-478 (2000)

54. Biskamp, D., Müller, W.C.: Scaling properties of three-dimensional isotropic magnetohydrodynamic turbulence. Phys. Plasmas 7(12), 4889-4900 (2000)

55. Müller, W.C., Biskamp, D.: The evolving phenomenological view on magnetohydrodynamic turbulence. In: Physics Lecture NotesNew York Then Berlin, vol. 614, pp. 3-27 (2003)

56. Müller, W.C., Biskamp, D., Grappin, R.: Statistical anisotropy of magnetohydrodynamic turbulence. Phys. Rev. E 67(6), 0306045 (2003)

57. Merrifield, J.A, Müller, W.C., Chapman, S.C., Dendy, R.O.: The scaling properties of dissipation in incompressible isotropic three-dimensional magnetohydrodynamic turbulence. Phys. Plasmas 12(2), 4889-4900 (2005)

58. Cho, J.Y., Lazarian, A., Vishniac, E.T.: Simulations of magnetohydrodynamic turbulence in a strongly magnetized medium. Astrophys. J. 564(1), 291-301 (2002)

59. Cho, J.Y., Lazarian, A.: Compressible magnetohydrodynamic turbulence: mode coupling, scaling relations, anisotropy, viscositydamped regime and astrophysical implications. Monthly Not. Roy. Astron. Soc. 345(1), 325-339 (2003)

60. Lazarian, A., Cho, J.Y.: Scaling, intermittency and decay of MHD turbulence. Phys. Scripta T 116, 32-37 (2005)

61. Cho, J.Y., Lazarian, A., Vishniac, E.T.: Ordinary and viscositydamped magnetohydrodynamic turbulence. Astrophys. J. 595(2), 812-823 (2003)

62. Gomez, T., Politano, H., Pouquet, A.: On the validity of a nonlocal approach for MHD turbulence. Phys. Fluids 11(8), 22982306 (1999)

63. Kowal, G., Lazarian, A.: Scaling relations of compressible MHD turbulence. Astrophys. J. 666(2), L69-L72 (2007)

64. Fang, L.Z.: Intermittency of cosmic baryon fluid. AIP Conf. Proc. 1059, 129-140 (2008)

65. He, P., Liu, J.R., Feng, L.L., Shu, C.W., Fang, L.Z.: Low-redshift cosmic baryon fluid on large scales and She-Leveque universal scaling. Phys. Rev. Lett. 96(5), 051302 (2006)

66. Liu, J.R., Fang, L.Z.: Non-Gaussianity of the cosmic baryon fluid: Log-Poisson hierarchy model. Astrophys. J. 672(1), 11-18 (2008)

67. Lu, Y., Chu, Y.Q., Fang, L.Z.: Log-poisson hierarchical clustering of cosmic neutral hydrogen and ly alpha transmitted flux of qso absorption spectrum. Astrophys. J. 691(1), 43-53 (2009)

68. Boldyrev, S.: Kolmogorov-Burgers model for star-forming turbulence. Astrophys. J. 569(2), 841-845 (2002)

69. Padoan, P., Boldyrev, S., Langer, W., Nordlund, A.: Structure function scaling in the Taurus and Perseus molecular cloud complexes. Astrophys. J. 583(1), 308-313 (2003)

70. Gustafsson, M., Brandenburg, A., Laire, J.L., Field, D.: The nature of turbulence in $\mathrm{OMC} 1$ at the scale of star formation: observations and simulations. Astron. Astrophys. 454(3), 815-825 (2006)

71. Avillez, De.M.A., Breitschwerdt, D.: The generation and dissipation of interstellar turbulence: results from large-scale highresolution simulations. Astrophys. J. 665(1), L35-L38 (2007) 
72. Uritsky, V.M., Paczuski, M., Davila, J.M., Jones, S.I.: Coexistence of self-organized criticality and intermittent turbulence in the solar corona. Phys. Rev. Lett. 99, 025001 (2007)

73. Kissmann, R., Kleimann, J., Fichtner, H., Grauer, R.: Local turbulence simulations for the multiphase ISM. Monthly Not. Roy. Astron. Soc. 391(4), 1577-1588 (2008)

74. Boldyrev, S., Nordlund, A., Padoan, P.: Scaling relations of supersonic turbulence in star-forming molecular clouds. Astrophys. J. 573(2), 678-684 (2002)

75. Padoan, P., Jimenez, R., Nordlund, A., Boldyrev, S.: Structure function scaling in compressible super-Alfvénic MHD turbulence. Phys. Rev. Lett. 92(19), 191102-191102 (2004)

76. Pan, L., Padoan, P., Kritsuk, A.G.: Dissipative structures in supersonic turbulence. Phys. Rev. Lett. 102(3), 034501 (2009)

77. Kritsuk, A.G., Norman, M.L.: Scaling relations for turbulence in the multiphase interstellar medium. Astrophys. J. 601(1), L55L58 (2004)

78. Efrov, Y.N., Elmegreen, B.G., Hodge, P.W.: Giant shells and stellar arcs as relics of gamma-ray burst explosions. Astrophys. J. Lett. 501(2), 163-165 (1998)

79. Frisch, U.: Turbulence. Cambridge University Press, Cambridge (1995)

80. Frisch, U., Sulem, P.L., Nelkin, M.: A simple dynamical model of intermittent fully developed turbulence. J. Fluid Mech. 87(4), 719-736 (1978)

81. Meneveau, C., Sreenivasan, K.R.: Simple multifractal cascade model for fully developed turbulence. J. Fluid Mech. Phys. Rev. Lett. 59(4), 1424-1427 (1974)

82. Benzi, R., Paladin, G., Parisi, G., Vulpiani, A.: On the multifractal nature of fully developed turbulence and chaotic systems. J. Phys. A 17, 3521-3531 (1984)

83. Guo, H.Y., Li, L., Qi, Q.Y., Liu, J., She, Z.S.: A systatic study of spirals and spiral turbulence in a reaction-diffusion syst. J. Chem. Phys. 118(11), 5038-5044 (2003)

84. Queiros-Conde, D.: Geometrical extended self-similarity and intermittency in diffusion-limited aggregates. Phys. Rev. Lett. 78(23), 4426-4429 (1997)

85. Turiel, A., Mato, G., Parga, N., Nadal, J.P.: Self-similarity properties of natural images resble those of turbulent flows. Phys. Rev. Lett. 80(5), 1098-1101 (1998)

86. Turiel, A., Parga, N., Ruderman, D.L., Cronin, T.W.: Multiscaling and information content of natural color images. Phys. Rev. E 62(1), 1138-1148 (2000)
87. Wang, J., Zhang, Q.D., Ren, K., She, Z.S.: Multi-scaling hierarchical structure analysis on the sequence of E-coli complete genome. Chin. Sci. Bull. 46(23), 1988-1992 (2001)

88. Ouyang, Z.Q., Wang, C., She, Z.S.: Scaling and hierarchical structures in DNA sequences. Phys. Rev. Lett. 93(7), 078103 (2004)

89. Ouyang, Z.Q., Liu, J.K., She, Z.S.: Hierarchical structure analysis describing abnormal base composition of genomes. Phys. Rev. E 72(4), 041915 (2005)

90. She, Z.S., Fu, Z.T., Chen, J., Liang, S., Liu, S.D.: Hierarchical structures in climate and atmospheric turbulence. Pro. Nat. Sci. 12(10), 747-752 (2002)

91. She, Z.S., Liu, L.: Measuring intermittency parameters of energy cascade in turbulence. Acta Mech. Sin. 19(5), 453-457 (2003)

92. Zhou, L.Q., Ouyang, Q.: Spiral instabilities in a reaction- diffusion syst. J. Phys. Chem. A 105(1), 112-118 (2001)

93. Winfree, A.T.: Electrical turbulence in three-dimensional heart muscle. Science 266(5187), 1003-1006 (1994)

94. Witkowski, F.X., Leon, L.J., enkoske, P.A., Giles, W.R., Spano, M.L., Ditto, W.L., Winfree, A.T.: Spatiotporal evolution of ventricular fibrillation. Nature(London) 392(6671), 78-82 (1998)

95. Turiel, A., Parga, N.: The multifractal structure of contrast changes in natural images: From sharp edges to textures. Neural Comput. 12(4), 763-793 (2000)

96. Grazzini, J., Turiel, A., Yahia, H., Herlin, I.: A multifractal approach for extracting relevant textural areas in satellite meteorological images. Environ. Model. Softw. 22(3), 323-334 (2007)

97. Ivanov, P.C., Amaral, L.A.N., Goldberger, A.L., Havlin, S., Rosenblum, M.G., Struzik, Z., Stanley, H.E.: Multifractality in human heartbeat dynamics. Nature 399, 461-465 (1999)

98. Ching, E.S.C., Lin, D.C., Zhang, C.: Hierarchical structure in healthy and diseased human heart rate variability. Phys. Rev. E 69, 051919 (2004)

99. Biskamp, D., Hallatschek, K., Schwarz, E.: Scaling laws in twodimensional turbulent convection. Phys. Rev. E 63, 045302 (2001)

100. Haugen, N.E.L., Brandenburg, A., Dobler, W.: Simulations of nonhelical hydromagnetic turbulence. Phys. Rev. E 70, 016308 (2004)

101. Cao, Y.H., Chen, J., She, Z.S.: The nature of near-wall convection velocity in turbulent channel flow. Acta Mech. Sin. 24, 587590 (2008) 